University of San Diego

Digital USD

Spring 5-31-2020

\title{
Enhancing Chronic Pain Management: Motivational Interview Among Lower Back Patients -Phase II
}

\author{
Valerie Gates \\ University of San Diego, vgates@sandiego.edu \\ Melissa Hines-Antico \\ University of San Diego, mhinesantico@sandiego.edu
}

Follow this and additional works at: https://digital.sandiego.edu/dnp

Part of the Nursing Commons

\section{Digital USD Citation}

Gates, Valerie and Hines-Antico, Melissa, "Enhancing Chronic Pain Management: Motivational Interview Among Lower Back Patients -Phase II" (2020). Doctor of Nursing Practice Final Manuscripts. 129. https://digital.sandiego.edu/dnp/129

This Doctor of Nursing Practice Final Manuscript is brought to you for free and open access by the Theses and Dissertations at Digital USD. It has been accepted for inclusion in Doctor of Nursing Practice Final Manuscripts by an authorized administrator of Digital USD. For more information, please contact digital@sandiego.edu. 


\title{
UNIVERSITY OF SAN DIEGO \\ Hahn School of Nursing and Health Science \\ DOCTOR OF NURSING PRACTICE
}

Enhancing Chronic Pain Management: Motivational Interview Among Lower Back Patients -

Phase II

by

Valerie N. Gates, BSN, RN

A Doctor of Nursing Practice Portfolio presented to the

FACULTY OF THE HAHN SCHOOL OF NURSING AND HEALTH SCIENCE

UNIVERSITY OF SAN DIEGO

\author{
In partial fulfillment of the \\ requirements for the degree \\ DOCTOR OF NURSING PRACTICE
}

May / 2020 


\title{
Final Manuscript
}

Enhancing Chronic Pain Management: Motivational Interview Among Lower Back Patients Phase II

\author{
Valerie N. Gates, BSN, RN \\ Melissa L. Hines-Antico, DNP, FNP \\ Joseph F. Burkard, DNSc, CRNA \\ University of San Diego
}




\begin{abstract}
Purpose: Chronic low back pain in adults is difficult to manage and can lead to clinical, psychological, and social consequences. Providers often have restricted clinic time that prevents patient education regarding self-care and non-pharmacological therapies.

Design: This nurse practitioner-led, evidence-based project incorporates monthly telephone calls to improve patient pain, enhance quality of life, decrease opioid usage, increase physical activity, and promote the utilization of non-pharmacological multimodal therapies.
\end{abstract}

Methods: Implementing a monthly telephone call utilizing motivational interviewing and a 5 A's guided questionnaire assisted in providing individualized treatment plans. Data outcomes include pain scores, quality of life scores, number of opioid pain medications, amount of intentional physical activity, and utilization of non-pharmacological multimodal therapies.

Results: Quality of life scores improved with each phone call and were not affected by pain medications. Higher pain scores were reported when taking one opioid medication. The most common non-pharmacological therapies used by patients were thermotherapy (heat/ice) and exercise but did not contribute to lower pain scores.

Conclusion: Chronic low back pain is difficult to treat and often requires a variety of modalities to improve function. The utilization of telephone calls was a viable and cost-effective method of interaction with patients that promoted health care access and patient education.

Clinical Implications: Utilizing the 5 A's behavior change model, motivational interviewing techniques, and monthly telephone calls to patients resulted in improved patient-reported quality of life scores, increased patient knowledge of non-pharmacological therapies, improved patient treatment plans, and increased revenue for the pain management clinic. 


\section{Background of Clinical Problem}

Chronic low back pain (LBP) in adults is difficult to manage and can lead to clinical, psychological, and social consequences. Over $40 \%$ of the adult population experiences chronic or recurrent pain at some point in their life (Von Korff et al., 2016). Chronic pain is defined as "pain that occurs on a least half of the days for 6 months or more" (p. 1069), and high-impact chronic pain is defined as "persistent pain with substantial restriction of participation in work, social, and self-care activities for 6 months or more" (Von Korff et al., 2016, p. 1070). Chronic pain conditions cost the United States over $\$ 600$ billion each year in direct medical treatment and lost productivity (Institute of Medicine [IOM], 2011). In 2013, California had an estimated incidence rate of 28 per 100,000 employees with a work-related lower back disorder hospitalization that was paid for by workers' compensation (California Department of Public Health, n.d.).

Opioids, commonly prescribed to treat low back pain, can lead to overdose or opioid use disorder (Dowell et al., 2016). There is insufficient evidence to support the use of long-term opioid use for chronic pain outside of end-of-life care (Dowell et al., 2016). Psychologically, chronic pain is linked to anxiety and depression placing patients at risk for opioid dependency (Goesling et al., 2018). In 2017, there were a total of 70,237 drug overdose deaths and adults aged 25-54 years had the highest rate of drug-related overdose deaths in the United States (Hedegaard, et al., 2018). In California, prescription opioids were the leading cause of opioidrelated deaths in 2017 (National Institute on Drug Abuse, 2019). In San Diego County, there were 273 per 100,000 residents that had an unintentional prescription-drug-related death in 2017 (San Diego Prescription Drug Abuse Task Force, 2017). 
Providers often have limited clinic time preventing patient education regarding self-care and non-pharmacological therapies. Patients need to be instructed that there are a variety of other non-opioid related treatments available to treat chronic LBP. Due to the high cost of treating chronic pain and the growing need to educate chronic pain patients about non-pharmacological treatments, there is growing interest in telehealth. Telehealth can help chronic pain patients by increasing accessibility to health care providers, personalized care in home environment, and potentially reducing costs. By removing barriers in accessing clinical care, it may improve treatment adherence and self-management behaviors (Adamse et al., 2018).

\section{Purpose of Evidence-Based Project}

The foundational question for this evidence-based practice (EBP) project is: In adults with chronic lower back pain, does implementing a monthly motivational interview using the 5 A's framework telephone call for 9 months, compared to no monthly telephone call, result in decreased pain scores, decreased opioid consumption, increased quality of life (QOL) scores, increased physical activity, and promote the utilization of non-pharmacological multimodal therapies.

The Iowa Model of Evidence-Based Practice to Promote Quality Care provides guidance for implementing practice change that affects patient outcomes (Melnyk \& Fineout-Overholt, 2015). The Iowa Model provides a practical methodology for translating research into practice by identifying triggers, forming a team, gathering research and evidence, critiquing and synthesizing evidence, developing a plan, changing practice, monitoring results, and disseminating results (Melnyk \& Fineout-Overholt, 2015). The Iowa Model was chosen for this project because it is a validated framework that implements systematic, multistep processes to guide changes in a large health care system (Iowa Model Collaborative et al., 2017). 


\section{Evidence-Based Interventions}

\section{Literature Review}

A review of the literature was conducted to provide support for the project. The literature review utilized the following search engines: CINAHL, PubMed, Cochrane, National Institute of Health, and Centers for Disease Control and Prevention (CDC). Keywords used for the search engines included: low back pain, motivational interview, 5A's behavior change, telephone-based follow-up/interview/coaching, quality of life, multimodal, non-pharmacological, telehealth, and chronic pain. The keywords were combined utilizing Medical Subject Headings (MeSH) and, depending on the search engine utilized, yielded over 75 articles. Articles were narrowed to include only English, adult patients, published after 2011, and no animals. Results of the literature review included 15 articles and of these articles, five were evaluated for their strength of evidence using the John Hopkins Evidence Level and Quality Guide. Three articles were ranked level I (i.e., high-quality randomized control trials) and two articles were ranked as level IV (i.e., high-quality clinical practice guidelines).

\section{Monthly Telephone Call}

The project utilized a monthly telephone follow-up call to chronic pain patients as a cost effective and proactive follow-up method for patients. Monthly telephone calls to assess patients' pain and discussion of a pain treatment plan led to a $30 \%$ improvement in perceived pain in patients with chronic musculoskeletal pain (Kroenke et al., 2014). Proactive calling on behalf of the provider to the patient to discuss pain can serve as a reminder for the patient to continue exercising, make an appointment for an office visit, or schedule a procedure. Telephone followup calls have been shown to be an effective method of promoting behavior change and managing chronic pain. 


\section{Motivational Interview}

Motivational interviewing (MI) is a method of promoting behavior change through coaching using therapeutic communication (Dart, 2011). The four principles of MI are express empathy, support self-efficacy, develop discrepancy, and roll with resistance (Dart, 2011).

Discussing resistance with the patients is the most challenging but it can lead to new opportunities. MI has been used to create meaningful interactions with the provider and involves active participation by the patient to reduce pain and increase QOL (Vong et al., 2011). MI can help patients increase their physical abilities, self- perceived general health, and compliance in performing home exercise (Vong et al., 2011). Helping the patient to see that certain behaviors they exhibit can impact the pain can be quite challenging (Dart, 2011). MI is beneficial communication, connection, and interaction between the provider and patient.

\section{A's Behavior Change Model}

The 5 A's behavior change model—assess, advise, agree, assist, and arrange — can be used to promote behavioral counseling interventions (U.S. Preventive Services Task Force, 2019). This model provides the framework for promotion of self-care management (Figure 1). As a validated framework, the 5 A's has been utilized extensively for chronic conditions requiring behavior change such as weight loss and smoking cessation (Glasgow et al., 2006). The 5 A's model can be applied to primary care interventions for a variety of other behaviors (U.S. Preventive Services Task Force, 2019). 


\section{Figure 1}

\section{A's Behavior Change Model}

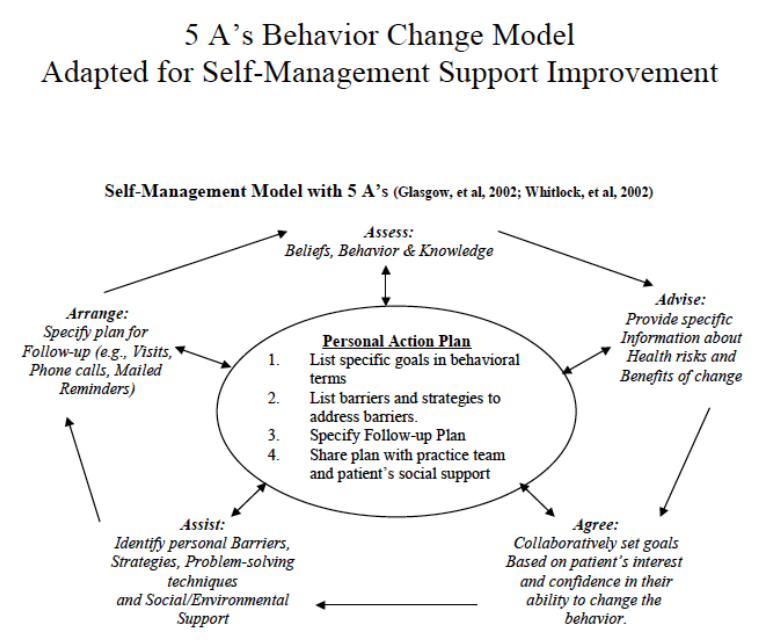

Note. Adapted from Behavioral counseling interventions: An evidence-based approach, by U.S.

Preventive Services Task Force, 2017.

(https://www.uspreventiveservicestaskforce.org/Home/GetFileByID/440)

\section{Individualized Treatment Plan}

Setting treatment goals is important when talking to patients about chronic pain. Goals should include improvements in pain relief, function, and QOL (Dowell et al., 2016). The American Society of Anesthesiologists (ASA Task Force on Chronic Pain Management, 2010) recommended a treatment plan that balances the risk-benefit ratio with an appropriate progression of least-to-most invasive forms of treatment. Direct and ongoing contact between provider and patient will promote individualized treatment plans. In chronic pain patients, it is essential to discuss that a pain-free state may not be attainable (ASA, 2010). In every provider encounter, patient goals and treatment plans should be addressed and updated. 


\section{Non-pharmacological Therapies}

There are a variety of other non-opioid related treatments available to treat chronic LBP. All patients should remain as active as possible and limit bedrest and sitting. The Centers for Disease Control and Prevention (CDC, 2016) recommends exercise, cognitive behavioral therapy, and interdisciplinary rehabilitation as non-pharmacological treatments for LBP. Multimodal therapies can reduce long term pain and disability compared with mere exercise alone. But multimodal therapies are not always available or reimbursed by insurance.

The practice guidelines for chronic pain management by the ASA strongly recommends using a multimodal approach when treating chronic pain. The guideline states that the "goal of treatment should be to effectively reduce pain while improving function and reducing psychosocial suffering" (ASA, 2010, p. 813). A multimodal approach—analgesics, selfmanagement strategies, and brief cognitive behavioral therapy—resulted in decreased pain severity and pain-related disability in patients with chronic musculoskeletal pain (Bair et al., 2015).

\section{Methods}

\section{Study Design}

The evidence-based quality improvement project was implemented at the University of California San Diego (UCSD) Center for Pain Management that serves as both a treatment facility for complex chronic pain conditions and provides consultation to primary care providers (PCP) for treatment plans. Members of the project team included two doctor of nursing practice (DNP) students as the project leads, one University of San Diego (USD) faculty advisor, two pain management specialists/anesthesiologists, and two medical assistants (MA). The project leads conducted all patient telephone calls, recording, and synthesis of data. All qualitative and 
quantitative data were recorded and dispersed through the clinics electronic health record (EHR) system.

Qualitative data was collected using a 5 A's guided questionnaire, entitled Personal action plan for chronic pain self-management, during the initial patient visit and with each monthly phone call (Appendix H).

Quantitative measurements in the project included the validated tools of (a) the numeric rating scale for pain (NRS), (b) the American Chronic Pain Association QOL scale, (c) the number of opioid pain medications, (d) amount of intentional physical activity daily, and (e) the use of any non-pharmacological therapies. The NRS for pain is used to measure pain intensity in adults, with $O$ representing no pain and 10 representing worst pain imaginable (Hawker et al., 2011). These tools were easily administered verbally or by telephone as well as being reliable and validated tools (Hawker et al., 2011).

The American Chronic Pain Association QOL Scale (Figure 2) helped patients and providers evaluate how pain was impacting basic activities of daily life (Cowan \& Kelly, 2003). The scale helped to measure activity levels, with 0 representing stay in bed all day feeling hopeless and helpless about life and 10 representing go to work/volunteer each day, have a social life, and take an active part in family life (Cowan \& Kelly, 2003). It was important for patients to understand that they might never be completely pain free and emphasized “encouraging self-management efforts to improve function and quality of life even if all pain cannot be eliminated" (IOM, 2011, p. 44). Measuring changes in function to assess pain may lead to better management of chronic pain rather than descriptions of pain. (IOM, 2011). 


\section{Figure 2}

\section{Quality of Life Scale}

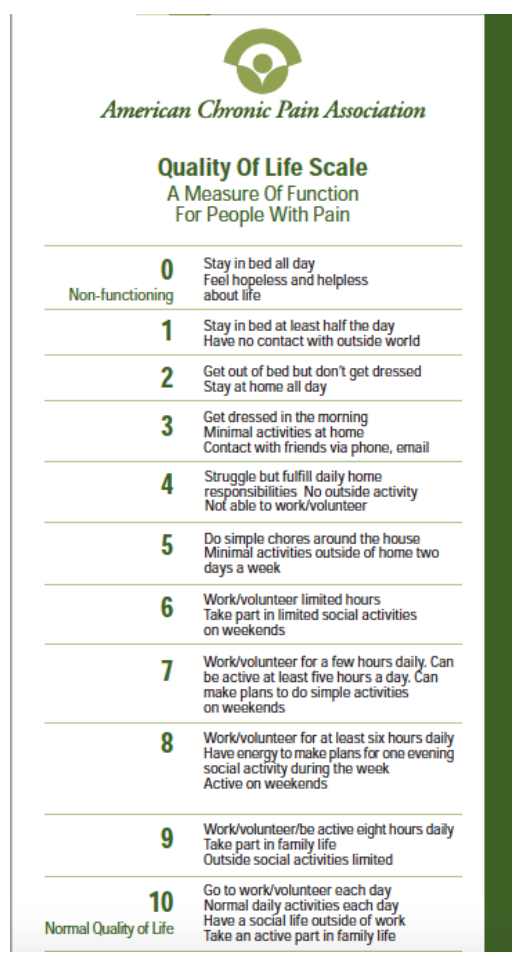

Note. Adapted from Quality of life scale, by American Chronic Pain Association, 2003. (https://www.theacpa.org/wp-content/uploads/2017/08/Life_Scale_3.pdf).

The number of opioid pain medications each patient was taking monthly was subjective data. None of the patients in this project were getting an opioid prescription from either of the anesthesiologists, but rather from their PCP. This was important data to collect because the goal was for the patient to reduce the amount of opioid medication needed after they received a procedure from the anesthesiologist. There was limited data on the effectiveness of opioid therapy for chronic back pain and opioids might even limit the effectiveness of other treatments (Deyo et al., 2015).

The amount of intentional physical activity daily was measured by asking the question, During the last month, on average have you been doing over thirty minutes of dedicated or 
intentional physical activity daily? The response was recorded as yes or no. A yes response was recorded if they attended physical therapy or performed any of the following: home physical therapy stretches, walking, or others form of physical activity. The U.S. Department of Health and Human Services (HHS, n.d.) recommended that adults should move more and sit less, but that could be difficult for patients with chronic LBP. Ultimately, all patients should try to be as physically active as their conditions will allow (HHS, n.d.).

The use of any non-pharmacological therapies was measured by asking the question, During the last month, have you used heat or ice, transcutaneous electrical nerve stimulation (TENS) unit, received a massage, acupuncture, or chiropractor treatment or anything else to help relieve your pain? The therapies used were recorded and logged as yes or no. A yes response was recorded if they used at least one non- pharmacological therapy as exercise, massage, yoga, acupuncture, spinal manipulation, and low-level laser therapy have been known to improve function and/or pain for at least 1 month after treatment (Skelly et al., 2018).

\section{Patient Demographics}

Patients were recruited from the two anesthesiologists' panel of patients. Inclusion criteria were patients over the age of 40, English speaking, and with chronic LBP (thoracic or lumbar), radiculopathy, and a recent low back invasive procedure. Exclusion criteria were patients whose primary language was not English, hearing difficulties, active cancer diagnosis, and actively receiving opioid medication from either anesthesiologist.

Phase I included 5 patients. All patients were Caucasian and ranged in age from 54 to 78 years with an average of 64 years. Four of the patients were male and 1 was female. All male patients were overweight with an average body mass index (BMI) of $26.4 \mathrm{~kg} / \mathrm{m}^{2}$. 
Phase II included 15 patients, including 4 patients from Phase I and 1 participant was lost to follow-up. Patients were aged 42 years to 92 years with an average of 66 years of age: $78 \%$ where Caucasian, 14\% where Asian, and 7\% where African American. A total of 9 females and 5 males participated. The average BMI was $26.8 \mathrm{~kg} / \mathrm{m}^{2}$.

\section{IRB Approval}

The EBP project, Motivation interview in follow-up telephone calls to pain patients to improve patient outcomes, was approved by UCSD Human Research Protections Program in October 2017. The EBP project was also approved by the Institutional Review Board (IRB) at USD in November 2017. Phase II DNP student, Valerie Gates, was added to the EBP project in November 2018 at USD's IRB and UCSD IRB with the assistance of Dr. Burkard, faculty advisor. No personal patient identifiers were used with any participant in the study. Patient identifiers were numbers known only to the project leads. There were no potential conflicts of interests or financial conflicts to disclose.

\section{Project Timeline}

Phase I of this EBP project began with a literature review and stakeholder presentation in September 2017 with Melissa Hines-Antico, project lead, Dr. Joseph Burkard, Timothy Furnish MD, and Gregory Polston MD. Pre-data collection was performed January 2018 to March 2018 followed by a 3-month period of analyzing the data to guide the project. Phase I data collection occurred between July 2018 to December 2018. The project transitioned to Valerie Gates between December 2018- January 2019. This transition process included introducing the new project lead to the stakeholders, recruiting and consenting new patients, and calling patients for the monthly telephone session. Phase I program evaluation was completed December 2018. Phase I results were presented to the stakeholders, along with a discussion about the future 
direction of this project in March 2019. Phase I dissemination of results occurred at the California Association for Nurse Practitioners and Western Institute of Nursing conferences March 2019 to April 2019 by the Phase I project lead.

Phase II data collection occurred between January 2019 and September 2019. Data analysis and program evaluation were completed by December 2019. The Phase II stakeholder presentation occurred in February 2020. Phase II dissemination of results happened in March 2020 at USD's DNP Presentation day. This EBP project was accepted to the California Association for Nurse Practitioners in September 2019 but could not be presented in March 2020 due to COVID-19.

\section{Project Implementation}

\section{Phase I}

Pre-data collection was gathered from January 2018 to March 2018 prior to project implementation and provided demographics of the general population within the pain clinic. The project lead accompanied the anesthesiologist during their scheduled clinic time to assess each patient. The patients' pain score, QOL score, demographics, type of pain, treatment plan, and follow-up were recorded. Out of 82 patients, $40 \%$ visited for LBP. Most patients are females between the ages of 60 years and 80 years with a BMI greater than 25 . The top two disturbances in patient-perceived QOL included sleep and exercise. Only 29\% of the patients had a solidified follow-up regimen, and $80 \%$ of the patients needed to schedule another appointment after their unplanned pain procedures. Patient diagnoses included LBP, LBP with radiculopathy, lumbar facet arthropathy, spinal stenosis of lumbar region, and lumbar spondylosis. Analysis of this preliminary data indicated that LBP should be the focus population. 
Data collection began July 2018 with reviewing the anesthesiologists' patient panels for eligible patients. Selected patients were seen for an office visit with the project lead and anesthesiologist. The anesthesiologist performed a health history, physical exam, assessment, and plan with the patient. At the end of the visit, the project lead provided the patient with an information sheet detailing the EBP project details. If the patient agreed to monthly phone calls, then verbal consent was obtained. The best contact phone number, day of the week, and time of day for calls was requested. Data points that were recorded during this visit including pain score using the NRS, QOL score, and number of pain medications currently taking.

During the next 5 months, the project lead reviewed each patient's EHR chart prior to calling to see if they had any procedures, office visits, or other events in the past month. Using the 5 A's guided questionnaire, the project lead called each patient and used MI techniques. The project lead used a list of MI open-ended questions to assess the patient's readiness to change a behavior. Assessing the patient's goals related to their pain and QOL helped to guide the conversation. Data points were recorded including pain score and goal pain score using the NRS, QOL score, and number of pain medications currently taking. At the conclusion of each telephone session, the project lead asked for consent to call again next month. All data were entered into the patient's EHR chart as a telephone note and sent to the primary anesthesiologist.

If the patient did not answer their phone, then a voicemail was left with a call-back number. If the patient did not return the call that day, the project lead called two additional times to reach the patient. If the project lead was unable to speak to the patient, then data were not collected. By having the project lead call these patients each month, there was an assessment to determine if the previous procedure was successful or unsuccessful. If patients had any concerns, the project lead was able to assist the patients in making an office visit, providing the procedure 
scheduling center phone number, or sending secure messages to the provider or MA through the EHR. Both project leads worked together December 2018 to January 2019 to consent new patients, make monthly phone calls, and write the telephone notes in the EHR chart.

\section{Phase II}

Phase II was a continuation of Phase I with modifications. The project lead continued to consent new patients until April 2019. One patient was lost to follow-up due to the inability to make contact over the phone after two consecutive months. The patient was withdrawn from the project and data were not included in this project. The provider was also notified.

Phase II included two additional data points on the Personal Action Plan for Chronic Pain Self-Management questionnaire: daily physical activity and use of any non-pharmacological therapies. If the patient responded no to either question, then the project lead would ask additional questions to evaluate the patient's barriers using MI techniques. If patients had questions about accessing non-pharmacological therapies (e.g., massage, acupuncture, chiropractic), the project lead did additional queries to give them information at the next phone call. Secure messages were sent to the providers and MAs to help schedule appointments, when procedures were not scheduled due to insurance issues, and each monthly telephone note.

\section{Data Analysis}

Phase I data included patient pain scores, QOL scores, and the number of opioid pain medications. Overall, the results suggested an increase in QOL scores and a decrease in pain scores among chronic LBP patients over 6-months. The average NRS pain score improved from 4.6 down to 3.8, almost a 1-point decrease. The average QOL score had the most dramatic increase from 4.6 to 6.0 signifying a change of scale scores from: Being able to do simple chores around the house and minimal activities outside of the home two hours a week to Work/volunteer 
limited hours and take part in limited social activities on weekends (Cowan \& Kelly, 2003). Pain medication increased by 0.2 , with 3 patients taking at least 1 opioid medication. One patient was prescribed two opioid medications after undergoing a major surgical procedure. The patient had a plan for dose reduction and a discontinuation date. Four out of five patients completed all six monthly-telephone follow-ups with only one patient missing 1 month due to a change in providers outside of this health care system.

Phase II data included patient pain scores, QOL scores, number of opioid pain medications, amount of daily physical activity, and the use of non-pharmacological therapies. Pain scores alone did not change over the course of Phase II. QOL scores suggested improvement with each phone call (Figure 3). On average, patients under 65 years of age reported higher pain scores and higher QOL scores then patients over 65 years of age. On average, women reported higher pain scores and lower QOL scores than men did. QOL scores were not affected by number of pain medications. Higher pain scores were reported when taking one opioid medication. The most common non-pharmacological therapies used by patients were thermotherapy (heat/ice) and exercise, but they did not contribute to lower pain scores (Figure 4). Pain scores and QOL scores did not appear to correlate with BMI, physical activity, or multimodal therapies; however, the sample size was not large enough for adequate power or to have any expectation of significant findings. 


\section{Figure 3}

Quality of Life Scores with Successive Telephone Calls

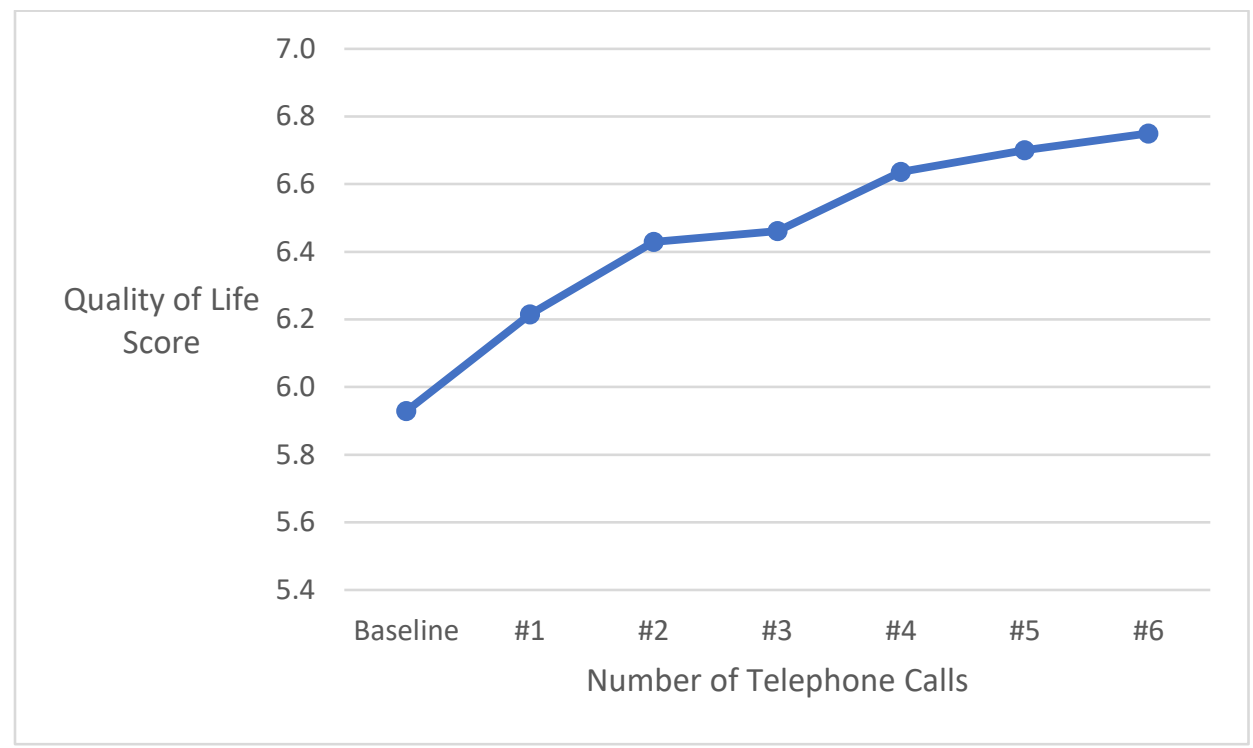

\section{Figure 4}

Number of Non-Pharmacological Therapies

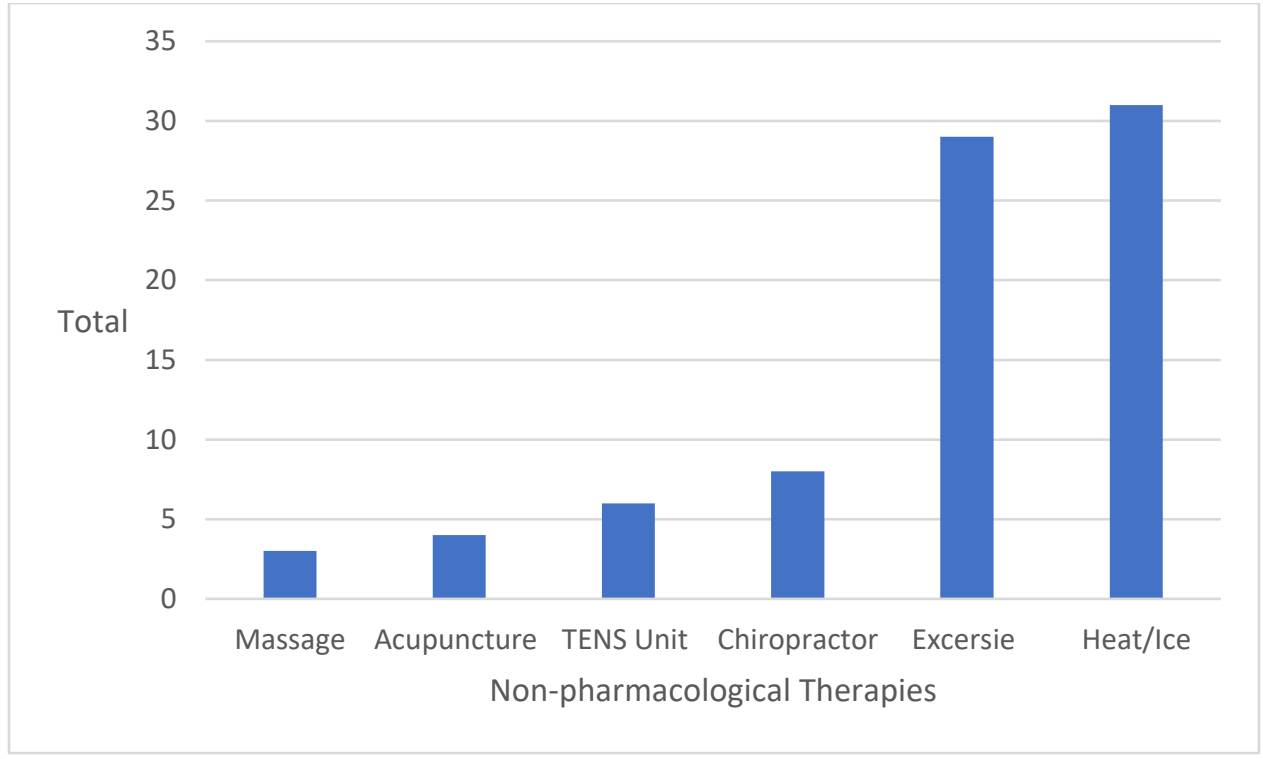




\section{Evaluation of Results}

Chronic LBP is difficult to treat due to inadequate treatments or medications that allow patients instant or lasting relief. This EBP project supported alternatives for managing chronic LBP. Phase I data indicated that a monthly telephone call using the 5 A's behavior change model and MI techniques improved patient reported pain scores and QOL scores. The intervention was meaningful and highlighted positive differences when patients were supported and able to make self-changing behaviors. Data revealed that pain scores peaked October 2018 and then drastically declined in December 2018. This could be attributed to an increase need for procedural interventions prior to the holiday season as outlined in patient interviews. Patients also noted an increase in stress prior to the holidays; one patient had major back surgery in 2018 that led to high levels of pain but improved over 3 months. This patient was briefly taking two opioid medications but had a planned opioid taper in place. QOL scores reflected patients' appreciation of a telephone call and feelings of individualized treatment. Patients were eager to discuss efficacy of procedures and how they utilized self-care such as exercise, improved sleep, alternate therapies, or overall sense of wellbeing.

Since Phase I demonstrated positive results, this project was expanded to Phase II. The stakeholders were pleased to continue the project with a second DNP student willing to assume the position of project lead. Phase II results showed similar results in QOL scores; improvement with each phone call. QOL scores where subjective to the project lead as patients did not have a copy of the American Chronic Pain Association QOL scale. The project leads asked questions about how often they left the house, how they spent their day, and if pain interfered with any activities of daily living. Based on these answers, the project lead assessed the QOL score. 
During Phase II, none of the patients were taking more than one opioid medication at a time. All opioids were prescribed by the patient's PCP. The anesthesiologists provided a lowback invasive procedure to patients every 3 months, at the patient's request. For most of the patients, this provided significant pain relief and they were able to stop taking their opioid medications. If the patients were not taking opioids, they were taking nonsteroidal antiinflammatory drugs, Tylenol, or lidocaine patches to help control their pain. It is not unexpected that higher pain scores were reported in patients taking opioid medication.

Although physical activity did not affect pain and QOL scores, it was thought to be an important part of helping the patients set goals. Most patients expressed that their chronic LBP limited their mobility. The goals that patients set for themselves typically had to do with improving their mobility or physical activity. The project lead asked each month if they had been working toward their mobility goal. If not, then there would be a discussion about barriers and how to overcome those barriers. The patient's mobility usually improved after getting a low-back invasive procedure; therefore, they had increased QOL, decreased pain, and more energy to do physical activity. One patient had a myocardial infraction in January 2019 and consequently had decreased mobility and increased LBP pain as bed rest was ordered for an extended period. Another patient did too much daily physical activity and frequently experienced muscle spasms and soreness. The monthly phone call also served as a reminder for patients to continue working on their mobility and physical activity goals.

The use of any non-pharmacological therapy did not appear to affect the patients pain or QOL scores; nevertheless, patients began to ask questions about other treatment options. Multimodal treatments have been categorized into five categories: procedural, pharmacological, 
psychological, physical, and other. As all patients received a recent low back invasive procedure, they were taking some form of pain medication (pharmacological).

A psychological assessment was deferred for this EBP project. UCSD pain management clinic recently hired a psychologist who specializes in pain management.

Physical activity comes in many forms and includes physical therapy, stretching/yoga, low-impact cardio, muscle strengthening, and walking. Patients who did any of these activities intentionally for 30 minutes daily were applauded for their effort. The category other included massage, acupuncture, thermotherapy (heat/ice), and a transcutaneous electrical nerve stimulation (TENS) unit. Many of the patients had tried these modalities in the past with no relief or were utilizing them to help control their pain. No one non-pharmacological therapy is considered superior to another and is a patient preference. Some patients requested information about certain non-pharmacological treatments. The project lead found resources in the community and shared that information with the patient during the subsequent telephone call.

Phase II results were collected during the summer of 2019, a common time for vacations, and were unable to speak to the project lead. One patient had a month-long trip Ireland and, having difficulty with sitting for long periods, was worried about the long flight. With the help of the project lead, they were able to get a lower epidural steroid injection before the trip. Overall patients seemed to enjoy the proactive telephone calls. The patients were engaged when planning their goals and discussing how they felt about their chronic LBP. Phase I and Phase II data indicated that a monthly telephone call using the 5 A's behavior change model and MI techniques improved a patient's reported QOL scores. 


\section{Cost-Benefit Analysis}

As this was a student-run DNP project, there were no costs associated with this project. The cost of paper and printer ink to provide patients with the project information handout was minimal. The project leads used the telephones and computers onsite. All patients in this project received a recent invasive low-back procedure. The most common procedures performed by the anesthesiologists were lumbar epidural steroid injections, radiofrequency ablations, trigger point injections, and sacroiliac injections. Most of these procedures can be repeated every 3 months. The patient is responsible for scheduling when they are ready for their next procedure. This can be problematic as patients forget to make the follow-up phone call, the patient's insurance does not approve the procedure, or the patient is unsure about the plan of care. These problems can cause patients to be lost to follow-up, to not receive a procedure due to insurance issues, and to wait for another referral from their PCP to return to the clinic. These issues also result in lost revenue for the pain management clinic.

With each monthly telephone call, the project leads were evaluating how effective the patient's previous procedure was and how they were currently feeling. An individualized treatment plan included a discussion about whether the low back procedure was helping the pain and if they wanted to continue with procedures every 3 months. The project lead also ensured that each patient had a follow-up plan, another procedure or an office visit, and if their pain was managed. While the project leads were unable to schedule procedure or office visits, they were able to send secure messages to the MA and provide the phone number for the procedure scheduling center. Lack of timely insurance approvals could also result in the project leads sending secure messages to the anesthesiologists and to an administrative assistant for help in 
securing procedure approved. These interventions leading to a scheduled office visit or procedure were recorded as an appointment.

During Phase I, two office visits where scheduled after the project lead telephoned the patient. Phase II resulted in 16 appointments created after speaking with the patients; 9 appointments for a low back invasive procedure and 7 return office visits. Table 1 below lists the current procedural terminology (CPT) codes and reimbursements according to the CDC (n.d.a; n.d.b). The estimated total revenue generated by Phase II of this EBP project was $\$ 6,012$.

Table 1

Total Appointments and Revenue Generated

\begin{tabular}{lcccc}
\hline & CPT Code & $\begin{array}{c}\text { Estimated } \\
\text { Payment }\end{array}$ & $\begin{array}{c}\text { Appointments } \\
\text { Created }\end{array}$ & $\begin{array}{c}\text { Total } \\
\text { Revenue }\end{array}$ \\
\hline $\begin{array}{l}\text { Lower } \\
\text { epidural } \\
\text { steroid } \\
\text { injection }\end{array}$ & 62323 & $\$ 598$ & 9 & $\$ 5,382$ \\
$\begin{array}{l}\text { Office visit - } \\
\text { established } \\
\text { patient }\end{array}$ & 99214 & $\$ 90$ & 7 & $\$ 630$ \\
$\begin{array}{l}\text { Project cost } \\
\quad \text { Total }\end{array}$ & & & & \\
\hline
\end{tabular}

Note. Estimated payments per CDC (n.d.a; n.d.b).

UCSD currently has telehealth capabilities, is accredited as a Clear Health Quality Institute, and can provide consumer-to-provider, provider-to-consumer, and provider-to-provider telehealth. Reimbursement for telehealth at UCSD is achieved through contracts with participating clinics. Providers using telehealth afford consults but not prescribe medications. Telehealth is not utilized by the pain management clinic at UCSD, but the continuation of the EBP project could assess the ability to be reimbursed directly for telephone calls to patients. 


\section{Implications for Clinical Practice}

Utilizing the 5 A's behavior change model, MI techniques, and monthly telephone call to patients resulted in improved patient reported pain and QOL scores, elevated patient knowledge of non-pharmacological therapies, more comprehensive patient treatment plans, and increased revenue for the pain management clinic. Patients were able to define and evaluate their own goals in achieving pain relief. Promoting self-care behaviors created a sense of independence for the patients. Monthly telephone calls fostered a stronger patient-provider relationship and led to more time spent with the providers. By having a structured follow-up plan, patients were able to work toward their mobility goals and have a greater QOL. The results of this project suggest that providers could improve patient outcomes with administrative time or telehealth visits to make telephone calls to patients with chronic LBP.

\section{Limitations/Sustainability}

This EBP project had limitations. Phase I had a small sample size that was increased during Phase II. This project was performed in a wealthy urban area that primarily serves older, Caucasian adults. Feasibility of this project should also be assessed within rural and minority populations. Telephone calls consumed more than the allotted 15 minutes causing delays in telephone calls to other patients. QOL scores were subjective and subject to project lead bias.

Recommendations for future projects would include the use of pain intensity, enjoyment of life, general activity (PEG) score to estimate pain and function, evaluate the use of non-opioid medications, and assess for behavior treatments, including cognitive behavioral therapy (USPSTF, 2019).

The UCSD pain management clinic does not employ any nurse practitioners, nurses, or case managers to assist with this patient population, but do employ two physician assistants. The 
clinic could benefit from additional personnel who would assist in making phone calls and connecting patients with other resources in the community to help manage their pain. The experience of seeing two DNP students in this clinic from January 2018 to February 2020 could lead to nurse practitioner hire in the future.

This EBP project was sustained at the UCSD pain management clinic for about two years. Without a nurse practitioner in this clinic it is unclear how this project will continue or be implemented into practice. Providers could ask for more administrative time to make telephone calls to patients and use the 5 A's behavior change model and MI techniques. A nurse, MA, or case manager could also make proactive phone calls to patients to discuss their chronic pain.

\section{Conclusion}

Chronic LBP is difficult to treat and often requires a variety of modalities to improve function. This EBP project implemented monthly telephone calls guided by the 5 A's framework to strengthen self-motivated behavior modifications, developed patient-centered outcomes through motivational interviewing, and promoted direct and ongoing communication between the provider and patient. This resulted in improved pain and QOL scores, increased patient knowledge of non-pharmacological therapies, and more comprehensive treatment plans. The utilization of telephone calls was a viable and cost-effective method of interaction with patients that promoteed health care access and patient education. This EBP project offers further insight into the importance of continued communication and the increased need for education regarding multimodal therapies among chronic LBP patients. 


\section{References}

Adamse, C., Dekker-Van Weering, M., van Etten-Jamaludin, F., \& Stuiver, M. M. (2018). The effectiveness of exercise-based telemedicine on pain, physical activity and quality of life in the treatment of chronic pain: A systematic review. Journal of Telemedicine and Telecare, 24, 511-526. https://doi.org/10.1177/1357633X17716576

American Society of Anesthesiologists Task Force on Chronic Pain Management. (2010). Practice guidelines for chronic pain management: An updated report by the American Society of Anesthesiologists Task Force on Chronic Pain management and the American Society of Regional Anesthesia and Pain Medicine Anesthesiology, 112(4), 810-833. https://doi.org/10.1097/ALN.0b013e3181c43103

Bair, M. J., Ang, D., Wu, J., Outcalt, S. D., Sargent, C., Kempf, C., Froman, A., Schmid, A. A., Damush, T. M., Yu, Z., David L. W., \& Kroenke, K. (2015). Evaluation of stepped care for chronic pain (ESCAPE) in veterans of the Iraq and Afghanistan conflicts: A randomized clinical trial. JAMA Internal Medicine, 175(5), 682-689. https://doi.org/10.1001/jamainternmed.2015.97

California Department of Public Health. (n.d.). California occupational health indicators: Annual measures of worker health and safety for years 2008-2013. Retrieved March 22, 2019 from https://www.cdph.ca.gov/Programs/CCDPHP/DEODC/OHB/Pages/Indicators.aspx

Centers for Medicare \& Medicaid Services. (n.d.a). Injection of substance into spinal canal of lower back or sacrum using imaging guidance. Retrieved March 25, 2019 from https://www.medicare.gov/procedure-price-lookup/cost/62323/ 
Centers for Medicare \& Medicaid Services. (n.d.b). Physician fee schedule. Retrieved March 25, 2019 from https://www.cms.gov/Medicare/Medicare-Fee-for-ServicePayment/PhysicianFeeSched/index

Centers for Medicare \& Medicaid Services. (2016). Nonopioid treatments for chronic pain. https://www.cdc.gov/drugoverdose/pdf/nonopioid_treatments-a.pdf

Cowan, P \& Kelly, N. (2003). The American Chronic Pain Association Quality of Life Scale. http://www.theacpa.org/wp-content/uploads/2017/08/Life_Scale_3.pdf

Dart, M. A. (2011) Motivational interviewing in nursing practice: Empowering the patient. Jones \& Bartlett.

Deyo, R. A., Von Korff, M., \& Duhrkoop, D. (2015). Opioids for low back pain. BMJ, 350, 113. https://doi.org/10.1136/bmj.g6380

Dowell, D., Haegerich, T. M., \& Chou, R. (2016). CDC guideline for prescribing opioids for chronic pain - United States, 2016. MMWR Recommendations and Reports, 65(1), 1-49. https://doi.org/10.15585/mmwr.rr6501e1

Glasgow, R. E., Emont, S., \& Miller, D. C. (2006). Assessing delivery of the five 'As' for patient centered counseling. Health Promotion International, 21(3), 245-255. https://doi.org/10.1093/heapro/dal017

Goesling, J., Lin, L. A., \& Clauw, D. J. (2018). Psychiatry and pain management: At the intersection of chronic pain and mental health. Current Psychiatry Reports, 20, 11-19. https://doi.org/10.1007/s11920-018-0872-4

Hawker, G. A., Mian, S., Kendzerska, T., \& French, M. (2011). Measures of adult pain: Visual Analog Scale for Pain (VAS Pain), Numeric Rating Scale for Pain (NRS Pain), McGill Pain Questionnaire (MPQ), Short-Form McGill Pain Questionnaire (SF-MPQ), Chronic 
Pain Grade Scale (CPGS), Short Form-36 Bodily Pain Scale (SF-36 BPS), and Measure of Intermittent and Constant Osteoarthritis Pain (ICOAP). Arthritis Care and Research, 63(Suppl. 11), S240-S252. https://doi.org/10.1002/acr.20543

Hedegaard, H., Minino, A. M., \& Warner, M. (2018). Drug overdose deaths in the United States, 1999-2017 [NCHS Data Brief, no 329]. https://www.cdc.gov/nchs/products/databriefs/db329.htm

Institute of Medicine. (2011). Relieving pain in America: A blueprint for transforming prevention, care, education, and research. National Academics Press.

Iowa Model Collaborative, Buckwalter, K.C., Cullen, L., Hanrahan, K., Kleiber, C., McCarthy A., Rakel, G., Steelman, V., Tripp-Reimer, T., \& Tucker, S. (2017). Iowa models of evidence-based practice: Revisions and validation. Worldviews on Evidence-Based Nursing, 14(3), 175-182. https://doi.org/ 10.1111/wvn.12223

Kroenke, K., Krebs, E. E., Wu, J., Yu, Z., Chumbler, N. R., \& Bair, M. J. (2014). Telecare collaborative management of chronic pain in primary care. A randomized clinical trial. JAMA, 312(3), 240-248. https://doi.org/10.1001/jama.2014.7689

Melnyk, B., \& Fineout-Overholt, E. (2015). Evidence-based practice in nursing \& healthcare: A guide to best practice (3rd ed.). Wolters Kluwer Health.

National Institute on Drug Abuse. (n.d.). California opioid summary: Opioid-related overdose deaths. Retrieved March 22, 2019 from https://www.drugabuse.gov/drugsabuse/opioids/opioid-summaries-by-state/california-opioid-summary

San Diego Prescription Drug Abuse Task Force. (2017). The status of prescription drug and heroin abuse in San Diego County. Retrieved March 22, 2019 from https://www.sandiegorxabusetaskforce.org/local-statistics 
Skelly, A. C., Chou, R., Dettori, J. R., Turner, J. A., Friedly, J. L., Rundell, S. D., Fu, R., Brodt, E. D., Wasson, N., Winter, C., Ferguson, A. J. R. (2018). Noninvasive nonpharmacological treatment for chronic pain: A systematic review [Comparative Effectiveness Review No. 209]. Agency for Healthcare Research and Quality. https://doi.org/10.23970/AHRQEPCCER209

U.S. Department of Health and Human Services. (n.d.). Physical activity guidelines for Americans. https://www.hhs.gov/fitness/be-active/physical-activity-guidelines-foramericans/index.html

U.S. Preventive Services Task Force. (2019). Behavioral counseling interventions: An Evidencebased approach. https://www.uspreventiveservicestaskforce.org/uspstf/behavioralcounseling-interventions-evidence-based-approach

Von Korff, M., Scher, A. I., Helmick, C., Carter-Pokras, O., Dodick, D. W., Goulet, J., HamillRuth, R., LeResche, L., Porter, L., Tait, R., Terman, G., Veasley, C., \&Mackey, S. (2016). United States national pain strategy for population research: Concepts, definitions, and pilot data. Journal of Pain, 17(10), 1068-1080. https://doi.org/10.1016/j.jpain.2016.06.009

Vong, S. K., Cheing, G. L., Chan, F., So, E. M., \& Chan, C. C. (2011). Motivational enhancement therapy in addition to physical therapy improves motivational factors and treatment outcomes in people with low back pain: A randomized controlled trial. Archives of Physical Medicine and Rehabilitation, 92(2), 176-183. https://doi.org/10.1016/j.apmr.2010.10.016 